\title{
Should we give metformin to all men with CRPC?
}

$\mathrm{M}$ etformin, which is the most commonly prescribed drug for type 2 diabetes mellitus (T2DM), could have an important role as an adjunct to combination therapies for men with castration-resistant prostate cancer (CRPC), according to the authors of a recent study published in European Urology. "The key findings of this study are that metformin, an old, well-tolerated and inexpensive drug, has activity in the treatment of some patients with CRPC, with an additional favourable effect on metabolic parameters," says Silke Gillessen, who led the study.

Although T2DM is associated with an increased risk of various cancers, including those of the breast, urinary tract, liver, and female reproductive organs, data suggest that men with T2DM have a $14 \%$ lower risk of developing prostate cancer than healthy controls. One proposed mechanism to explain this association is an anticancer effect of some of the drugs used to treat T2DM, namely metformin and the thiazolidinediones, which have antiproliferative effects in preclinical models of prostate cancer. This theory is supported by a study of almost 4,000 men with T2DM and prostate cancer, which found that metformin use was associated with reduced prostate-cancer-specific and all-cause mortality.

Now, Rothermundt et al. have published the first prospective study of the effects

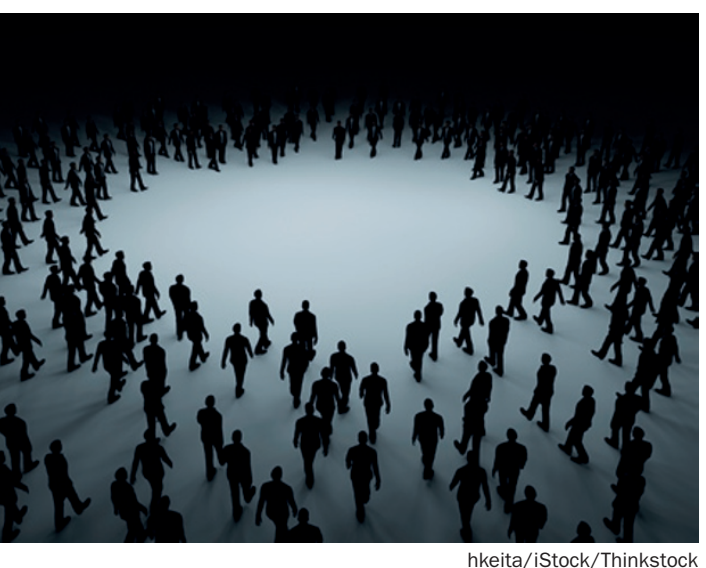

of metformin treatment in men with CRPC but no pre-existing T2DM. In this single-arm study involving 10 clinics in Switzerland, 44 chemotherapy-naive men with metastatic CRPC were given $1,000 \mathrm{mg}$ metformin twice daily until they experienced disease progression (defined as a PSA increase of $\geq 25 \mathrm{ng}$ above baseline, progression of measureable disease or bone lesions, clinical progression, or start of palliative radiotherapy). Disease status was assessed every 12 weeks with physical examination, CT of the chest, abdomen, and pelvis, bone scanning, PSA testing, and laboratory evaluation.

Overall, metformin was shown to have a modest anticancer activity in this cohort; $36 \%$ and $9 \%$ of these men were progression-free at 12 weeks and 24 weeks, respectively, PSA doubling time was slightly prolonged in $52 \%$ of patients (although this effect was not deemed to be statistically significant), and two patients ( $5 \%$ of the group) experienced a PSA decline of $\geq 50 \%$. Median progression-free survival (PFS) was 2.8 months and $46 \%$ of patients were deemed to have derived some clinical benefit from treatment. Importantly, all treatment-related adverse effects were mild and manageable, with no reports of grade 3 or 4 events.

Rothermundt and colleagues suggest several mechanisms (both direct and indirect) by which metformin might exert its anticancer effects. Firstly, this drug is likely to indirectly suppress prostate cancer progression by restoring insulin sensitivity, as insulin is known to increase intratumoral androgen production, driving disease progression. Metformin also has potential antineoplastic activity via direct activation of $\mathrm{p} 53$ and the AMP-kinase pathway, downregulation of cyclin D1, inhibition of the mTOR pathway, and suppression of HER2 oncoprotein expression.

So, in light of this anticancer activity, should we give metformin to all men with CRPC? Although it is too early for broad recommendations, these initial data suggest that such an approach should not be ruled out for the future. Not only is metformin relatively cheap, several studies have now shown that metformin is safe to use in men without diabetes. Thus, metformin is unlikely to harm the patient and could offer benefits, both in terms of disease progression and metabolic health (including reduced insulin levels and weight loss). Given that men receiving androgen deprivation therapy are at increased risk of developing insulin resistance, hyperglycaemia, and obesityfactors that are all associated with a shorter time to PSA progression and reduced overall survival-these metabolic benefits should not be undervalued.

However, before jumping the gun, it is important to remember that the findings of Rothermundt et al. are limited by several factors, including the small sample size and nonrandomized single-arm nature of their study. Indeed, the primary end point of PFS at 12 weeks could have been met by these patients in the absence of intervention, although the prominent PSA decline experienced by $5 \%$ of these men suggests that metformin does have some anticancer activity, even if only in a small subpopulation.

"This study supports the case for further research into the effects of metformin, both with other drugs and in earlier phases of disease," says Gillessen. "Discussions are underway with a large UK collaborative group to study metformin further in large prospective randomized trials. In Switzerland, we are testing a combination of metformin and abiraterone acetate in men who have progressed on abiraterone acetate."

\section{Melanie Clyne}

Original article Rothermundt, C. et al. Metformin in
chemotherapy-naive castration-resistant prostate cancer:
a multicenter phase 2 trial (SAKK 08/09). Eur. Urol.
doi:10.1016/j.eururo.2013.12.057
Further reading De Nunzio, C. \& Tubaro, A. Prostate cancer:
Diabetes and prostate cancer-an open debate. Nat. Rev.
Urol. 10,12-14 (2013)

